\title{
Association of variants in the fat mass and obesity associated (FTO) gene with polycystic ovary syndrome
}

\author{
T. M. Barber • A. J. Bennett • C. J. Groves • U. Sovio • \\ A. Ruokonen - H. Martikainen • A. Pouta • \\ A.-L. Hartikainen - P. Elliott • C. M. Lindgren • \\ R. M. Freathy $\cdot$ K. Koch $\cdot$ W. H. Ouwehand $\cdot$ F. Karpe • \\ G. S. Conway • J. A. H. Wass • M.-R. Järvelin • \\ S. Franks • M. I. McCarthy
}

Received: 26 January 2008 / Accepted: 8 April 2008 / Published online: 14 May 2008

(C) Springer-Verlag 2008

\begin{abstract}
Aims/hypothesis Variants in the fat-mass and obesityassociated gene (FTO) influence susceptibility to type 2 diabetes via an effect on adiposity/obesity. Given the important role of obesity in the aetiology of both polycystic ovary syndrome (PCOS) and type 2 diabetes mellitus, our aim was to establish whether FTO variants are also implicated in PCOS susceptibility.
\end{abstract}

T. M. Barber $(\triangle) \cdot$ A. J. Bennett $\cdot$ C. J. Groves $\cdot$ F. Karpe $\cdot$

J. A. H. Wass $\cdot$ M. I. McCarthy

Oxford Centre for Diabetes, Endocrinology and Metabolism,

Churchill Hospital,

Old Road, Headington,

Oxford OX3 7LJ, UK

e-mail: tom.barber@drl.ox.ac.uk

U. Sovio $\cdot$ P. Elliott $\cdot$ M.-R. Järvelin

Department of Epidemiology and Public Health, Imperial College (St Mary's Campus),

London, UK

\section{A. Ruokonen \\ Department of Clinical Chemistry, \\ Oulu University Hospital and University of Oulu, \\ Oulu, Finland}

H. Martikainen · A. Pouta - A.-L. Hartikainen

Department of Obstetrics and Gynecology,

Oulu University Hospital and University of Oulu,

Oulu, Finland

\section{A. Pouta}

National Public Health Institute,

Oulu, Finland

C. M. Lindgren $\cdot$ M. I. McCarthy

Wellcome Trust Centre for Human Genetics,

Oxford, UK
Methods We performed a genetic association study of FTO variant rs9939609 using case-control analyses, conducted in 463 PCOS patients (geometric mean BMI $27.5 \mathrm{~kg} / \mathrm{m}^{2}$ ) and 1,336 female controls (geometric mean BMI $25.3 \mathrm{~kg}$ / $\mathrm{m}^{2}$ ) of UK British/Irish origin. We also sought evidence for associations between FTO variation and circulating testosterone levels in 324 UK PCOS patients and 1,000 women from the Northern Finland Birth Cohort of 1966. Outcome

R. M. Freathy

Institute of Biomedical and Clinical Science,

Peninsula Medical School,

Exeter, UK

K. Koch • W. H. Ouwehand

Department of Haematology, University of Cambridge and National Health Service Blood and Transplant,

Cambridge, UK

\section{G. S. Conway}

University College Hospital,

London, UK

M.-R. Järvelin

Department of Public Health Science and General Practice, Oulu University Hospital and University of Oulu, Oulu, Finland

\section{S. Franks}

Institute of Reproductive and Developmental Biology, Imperial College (Hammersmith Campus),

London, UK 
measures included FTO rs9939609 genotype frequencies by participant group and androgen measures (testosterone, free androgen index) by genotype.

Results There was a significant association between FTO genotype and PCOS status in the UK case-control analysis, which was attenuated by adjustment for BMI (CochranArmitage test, odds ratio [per minor allele copy] 1.30 [95\% CI 1.12, 1.51], $p=7.2 \times 10^{-4}$ [unadjusted], $p=2.9 \times 10^{-3}$ [adjusted]). This association was most evident in obese PCOS patients (PCOS patients below median BMI vs UK controls, $p=0.11$; above median BMI vs controls, $p=2.9 \times$ $10^{-4}$ ). No relationship between FTO genotype and androgen levels was seen.

Conclusions/interpretation We provide the first evidence that variants that predispose to common obesity also result in altered susceptibility to PCOS, confirming the mechanistic link between these conditions. The predominant effect of FTO variants on PCOS susceptibility is probably mediated through adiposity.

Keywords Androgens . FTO variants · Obesity .

Polycystic ovary syndrome · Type 2 diabetes mellitus

$\begin{array}{ll}\text { Abbreviations } \\ \text { FAI } & \text { free androgen index } \\ \text { NFBC1966 } & \text { Northern Finland Birth Cohort of } 1966 \\ \text { OBB } & \text { Oxford Biobank } \\ \text { PCOS } & \text { polycystic ovary syndrome } \\ \text { SHBG } & \text { sex hormone binding globulin } \\ \text { UKBS } & \text { UK Blood Services } \\ \text { WTCCC } & \text { Wellcome Trust Case Control Consortium }\end{array}$

\section{Introduction}

Polycystic ovary syndrome (PCOS) is a common female endocrinopathy with a prevalence of between 5\% and 7\% in premenopausal women, and frequently co-exists with both obesity and type 2 diabetes mellitus [1-3]. Substantial evidence implicates obesity as an important factor in the aetiology of PCOS [4-7], though the mechanistic links between these conditions and how they interact with type 2 diabetes mellitus are poorly understood. Both obesity and PCOS are highly heritable [8-10], raising the possibility that a shared genetic predisposition contributes to their cooccurrence. However, until recently there has been little success in the identification of susceptibility genes for either condition [11, 12].

Recent work has shown that common variants in the fat mass and obesity associated gene (FTO) influence susceptibility to type 2 diabetes mellitus via a substantial effect on $\mathrm{BMI}$ and fat mass, establishing this as the first gene with a robust effect on individual risk of common polygenic obesity $[13,14]$. Though the causal variant has yet to be identified, the association signal maps to a $47 \mathrm{~kb}$ region in the first intron of FTO. In the UK, the strongest associations are seen for a cluster of highly correlated single-nucleotide polymorphisms (SNPs), of which rs9939609 is representative. These variants are associated with a median per-allele difference in BMI of approximately $0.36 \mathrm{~kg} / \mathrm{m}^{2}$ and susceptibility to type 2 diabetes mellitus (odds ratio 1.27) [13].

Given the effect of FTO on adiposity and susceptibility to the development of type 2 diabetes mellitus, the close relationship between obesity and PCOS, and the common pathophysiological and epidemiological features shared by type 2 diabetes mellitus and PCOS, we set out to establish whether FTO variants also impact on the individual risk of PCOS. We did so in the belief that detecting such an association would provide further evidence (complementing that from epidemiology and studies of weight loss intervention) that obesity and PCOS are causally related, as well as providing evidence that genome sequence variation can influence predisposition to PCOS. A further aim was to establish whether FTO variation influences circulating levels of testosterone in women with PCOS, given that hyperandrogenaemia is the most consistent endocrine feature and forms part of the diagnostic criteria for PCOS $[7,15]$.

\section{Methods}

Participants We studied 463 UK PCOS patients and 1,336 UK female controls. All UK European British/Irish patients had a confirmed diagnosis of PCOS according to the consensus Rotterdam criteria [15]. More specifically, all UK patients had ultrasound-confirmed polycystic ovarian morphology [16] in addition to a history of hyperandrogenism (defined as previously described, other aetiologies having been excluded) [17] and/or oligo-amenorrhoea (intermenstrual interval $>42$ days). The vast majority of PCOS patients $(n=459)$ had no known history of type 2 diabetes mellitus. All PCOS patients were non-pregnant, and were recruited from St Mary's and Middlesex (University College) Hospitals in London (as previously described) [18], or from Oxford-based endocrine clinics $(n=61)$ using the same ascertainment criteria.

Women from two UK population-based control groups (all of European British/Irish origin) were genotyped. These included 565 women in the Oxford Biobank (OBB), drawn from the UK National Health Service population register. Genotypes were also available from 771 women in the UK Blood Services (UKBS) controls, set up by the Wellcome Trust Case Control Consortium (WTCCC) in collaboration 
with the UK Blood Services. Height and weight measurements were recorded for all OBB female controls and for 769 UKBS control women (the latter self-reported).

We also examined the relationship between FTO genotype and androgen levels (as a continuous trait relevant to PCOS status) in 324 of the UK cases and in 1,000 women from the population-based Northern Finland Birth Cohort of 1966 (NFBC1966, described previously [6, 19-21]), in whom there were available measures of both testosterone and FTO genotype. None of these women was on oral hypoglycaemic agents (including metformin) or hormonal therapy, and none was pregnant at the time of study. In addition, none of the women examined from the NFBC1966 had described any symptoms of PCOS in a questionnaire completed at the age of 31 years.

Clinical details of all cases and control groups are shown in Table 1. Serum testosterone and sex hormone binding globulin (SHBG) concentrations were measured as previously described [18] and the free (unbound) androgen index (FAI) was calculated as total testosterone $(\mathrm{nmol} / \mathrm{l}) \times 100 /$ SHBG (nmol/1). All clinical investigations were conducted in accordance with the guidelines in the Declaration of Helsinki. All participants provided fully informed written consent and the study was approved by the relevant ethics committees in the UK and Finland.

Genotyping For the UK patients and NFBC1966 women, genotyping of FTO rs9939609 was performed using a Taqman assay on demand method (assay ID, C_30090620_10; Applied Biosystems, Warrington, UK). For the OBB control group, genotyping of FTO rs9939609 was performed by Kbiosciences (Hoddesdon, UK; http:// www.kbioscience.co.uk) using a fluorescence-based competitive allele-specific (KASPar) assay. For the UKBS control group, FTO rs9939609 genotypes were obtained from the Affymetrix GeneChip Human Mapping 500k Array Set as part of the WTCCC study, as previously reported [13]. We have established high concordance rates $(>99.8 \%)$ between rs9939609 genotypes for the same samples generated on these platforms, and $(>99.9 \%)$ for duplicate genotypes on the same (Taqman) platform. Genotype success rates exceeded $96 \%$ in all samples and there were no departures from Hardy-Weinberg equilibrium $(p>0.05)$. Details of all assays are available on request.

Statistical analyses and power calculations Genotype frequency comparisons were conducted using the CochranArmitage (additive) test (StatXact v.6; Cytel, Cambridge, MA, USA). Following appropriate distributional logarithmic transformations, one-way ANOVA (conducted in SPSS [v12.0; SPSS, Chicago, IL, USA]) was used for quantitative trait analyses (testosterone and FAI). Testosterone levels were optionally adjusted for BMI (where available). It is important to realise that, in the UK case-control analysis, the use of population-based controls (as opposed to controls in whom a diagnosis of PCOS has been excluded by clinical examination) results in only a modest loss of power and that this can be overcome easily (as here) by increasing the number of controls [22]. As there was no evidence of heterogeneity between genotype counts between women in the two UK control groups ( $p=0.91$ ), our primary UK casecontrol analyses were based on a comparison of cases with the (combined) female controls.

Power calculations were performed using Quanto v.0.5.5 (log-additive model) (http://hydra.usc.edu/GxE/, accessed April 2008). In the case-control analyses, the available UK sample sizes provided $91 \%$ power to detect an allelic odds ratio of 1.3 at an $\alpha$ value of 0.05 . For the Finnish (NFBC1966) women whose data were used for the quantitative trait (testosterone) analyses, we had $80 \%$

Table 1 Clinical characteristics of UK and Finnish NFBC1966 participants

\begin{tabular}{lllll}
\hline & UK PCOS patients & OBB female controls & UKBS female controls & NFBC1966 women \\
\hline Number & $463^{\mathrm{a}}$ & 565 & 771 & $1,000^{\mathrm{b}}$ \\
Age (years) & $32.3 \pm 7.0^{\mathrm{c}}$ & $41.5 \pm 6.3^{\mathrm{c}}$ & $41.6 \pm 12.7^{\mathrm{c}}$ & $31^{\mathrm{d}}$ \\
BMI $\left(\mathrm{kg} / \mathrm{m}^{2}\right)$ & $27.5(21.2,35.7)$ & $25.2(21.3,29.8)$ & $25.4(21.5,30.0)$ & $24.0(20.0,28.8)$ \\
WHR & $0.79(0.72,0.87)$ & $0.80(0.73,0.86)$ & Not known & $0.81(0.74,0.89)$ \\
Testosterone $(\mathrm{nmol} / \mathrm{l})$ & $2.1(1.4,3.1)^{\mathrm{e}}$ & Not known & Not known & $1.9(1.2,2.9)$ \\
Free androgen index & $6.0(2.9,12.4)^{\mathrm{e}}$ & Not known & Not known & $3.3(1.7,6.3)$ \\
Glucose $(\mathrm{mmol} / \mathrm{l})$ & $4.8(4.2,5.4)^{\mathrm{e}, \mathrm{f}}$ & $5.0(4.6,5.4)^{\mathrm{f}}$ & Not known & $4.9(4.3,5.6)^{\mathrm{f}}$ \\
\hline
\end{tabular}

Quantitative data are presented as geometric mean (SD range) unless otherwise stated. All participants were female

${ }^{a}$ UK non-pregnant PCOS patients

${ }^{\mathrm{b}}$ Excluding women on oral hypoglycaemic agents, metformin or hormonal therapy (oral contraception or hormonal intrauterine device), women pregnant at the time of examination and women with symptoms of PCOS described in the questionnaire completed at the age of 31 years ${ }^{\mathrm{c}} \mathrm{Mean} \pm \mathrm{SD}$

${ }^{\mathrm{d}}$ All women in the NFBC1966 were sampled at the age of 31 years

${ }^{\mathrm{e}}$ Excluding women on oral hypoglycaemic agents, metformin or hormonal therapy (oral contraception)

${ }^{\mathrm{f}}$ Fasting samples 
Table 2 Case-control association analyses for the relationship between variants at FTO rs9939609 and PCOS in UK samples

\begin{tabular}{|c|c|c|c|c|c|}
\hline & $n$ & $\mathrm{TT}$ & AT & AA & $p$ value vs PCOS patients ${ }^{\mathrm{a}}$ \\
\hline Alleles in UK PCOS patients & 463 & $133(28.7 \%)$ & $231(49.9 \%)$ & $99(21.4 \%)$ & \\
\hline \multicolumn{6}{|l|}{ Alleles in controls } \\
\hline OBB women & 565 & $204(36.1 \%)$ & $269(47.6 \%)$ & $92(16.3 \%)$ & $4.6 \times 10^{-3}$ \\
\hline UKBS women & 771 & $276(35.8 \%)$ & $375(48.6 \%)$ & $120(15.6 \%)$ & $1.7 \times 10^{-3}$ \\
\hline Combined women & 1,336 & $480(35.9 \%)$ & $644(48.2 \%)$ & $212(15.9 \%)$ & $7.2 \times 10^{-4}$ \\
\hline
\end{tabular}

Data shown are genotype counts (and percentages). A allele is associated with an increase in BMI. $p$ values represent Cochran-Armitage test results

${ }^{\text {a }}$ Comparison with UK PCOS patients

power to detect a trait difference between each genotype group (per minor allele copy) exceeding $25.1 \%$ of a standard deviation (for $\alpha=0.05$ ).

\section{Results}

Minor allele frequencies for FTO rs9939609 in the UK PCOS patients and combined female control groups were 46.3 and $40.0 \%$ respectively. Genotype frequency comparisons revealed a significant association of the minor (A) allele with PCOS (Cochran-Armitage test, odds ratio [per minor allele] $1.30,95 \%$ CI 1.12, 1.51, $p=7.2 \times 10^{-4}$; Table 2). The expected relationship between FTO genotype and BMI was observed in the UK patients (per allele difference in BMI $1.1 \mathrm{~kg} / \mathrm{m}^{2}, 95 \%$ CI $-0.9,3.2, p=0.05$ ), but less obvious in the controls (per allele difference in BMI $0.5 \mathrm{~kg} / \mathrm{m}^{2}, 95 \%$ $\mathrm{CI}-0.1,1.3, p=0.33$ ). Following adjustment for BMI in the comparison between UK patients and the combined control group, the association with PCOS was attenuated but not eradicated $\left(p=2.9 \times 10^{-3}\right)$. Separate subgroup analyses were performed in UK patients with known BMI $(n=430)$, stratified according to whether their BMI was above or below the median for the case group $\left(26.0 \mathrm{~kg} / \mathrm{m}^{2}\right)$. Minor allele frequencies for UK patients in the high- and low-BMI strata were 49.3 and $44.0 \%$ respectively, with only the former stratum significantly associated with PCOS when compared with the controls (high-BMI patients, $p=2.9 \times$ $10^{-4}$; low-BMI patients, $p=0.11$ ).

Analyses of testosterone and FAI between FTO rs9939609 genotype groups were conducted separately in UK patients $(n=324)$, and women from the NFBC1966 $(n=$ $1,000)$, following exclusion of those taking hormonal therapy, metformin or any other oral hypoglycaemic agents. No significant overall trend was shown for testosterone or FAI in either the UK patients or the NFBC1966 groups (Table 3).

\section{Discussion}

This study, involving $>1,700$ women from the UK and 1,000 from Finland, is the first to demonstrate that variation within the FTO gene is significantly associated with PCOS. Although the levels of significance attained are not conclusive on the

Table 3 FTO rs9939609 genotypes and analyses of androgen measures

\begin{tabular}{|c|c|c|c|c|c|c|}
\hline & TT & AT & $\mathrm{AA}$ & Total & $p$ value & $p$ value adjusted for BM \\
\hline \multicolumn{7}{|l|}{ UK patients } \\
\hline Number $^{\mathrm{a}}$ & 96 & 160 & 68 & 324 & & \\
\hline Testosterone (nmol/1) & $2.1(1.4,3.2)$ & $2.1(1.4,3.0)$ & $2.3(1.5,3.4)$ & & 0.31 & 0.75 \\
\hline FAI $^{\mathrm{b}}$ & $6.2(3.1,12.5)$ & $5.5(2.8,11.8)$ & $6.9(3.5,14.2)$ & & 0.24 & \\
\hline \multicolumn{7}{|l|}{ NFBC1966 } \\
\hline Number ${ }^{\mathrm{c}}$ & 375 & 453 & 172 & 1,000 & & \\
\hline Testosterone (nmol/1) & $1.9(1.3,2.9)$ & $1.8(1.2,2.8)$ & $1.8(1.2,2.9)$ & & 0.22 & 0.05 \\
\hline FAI $^{\mathrm{b}}$ & $3.5(1.7,6.2)$ & $3.5(1.7,6.2)$ & $3.5(1.7,6.6)$ & & 0.05 & \\
\hline
\end{tabular}

A allele is associated with an increase in BMI. Testosterone and FAI values are expressed as geometric mean (SD range). FAI was not adjusted for BMI, given the high correlation between BMI and SHBG concentration

${ }^{a}$ All UK PCOS British/Irish patients excluding women on oral hypoglycaemic agents (including metformin) or hormonal therapy

${ }^{\mathrm{b}}$ Free androgen index calculated as total testosterone $(\mathrm{nmol} / \mathrm{l}) / \mathrm{SHBG}(\mathrm{nmol} / \mathrm{l}) \times 100$

${ }^{\mathrm{c}}$ Excluding women on oral hypoglycaemic agents (including metformin), hormonal therapy, women pregnant at the time of examination and women with symptoms of PCOS described in the questionnaire completed at the age of 31 years 
genome-wide scale (which would require $p<10^{-7}$ ), the strong prior claims for FTO (given its incontrovertible association with fat mass and type 2 diabetes mellitus) [13, 14] make this one of the first convincing claims for a relationship between genome sequence variation and PCOS susceptibility.

It seems likely that the effect of FTO on PCOS susceptibility is mediated through its effect on fat mass and the risk of obesity, in a fashion analogous to the relationship between $F T O$ variation and predisposition to type 2 diabetes mellitus [13]. In line with previous studies (involving over 40,000 participants) [13, 14, 23], we found evidence that FTO variants influenced adiposity levels in our sample, though this effect was only nominally significant in the patients. Given previous large-scale studies that have shown no evidence for heterogeneity of FTO effect size between samples of diverse origin, including the same controls as used here [13], we regard the failure to detect a relationship between FTO rs9939609 and adiposity in the controls as entirely consistent with sampling error (indeed, the $95 \%$ CI range for the FTO effect in controls includes the mean effect size seen in previous papers) $[13,14,23]$.

In the present study, and in contrast to the situation in type 2 diabetes mellitus [13], the PCOS association was not fully abolished by adjusting for BMI. This may well reflect sampling error, and the inadequacy of a single measure of weight and height as an index of chronic excess adiposity. Evidence that the case-control association was far weaker when restricted to the leaner PCOS patients supports this interpretation. To the extent that adjustment for BMI diminishes the PCOS association, our data provide further evidence, complementing that from epidemiology and the effects of weight reduction on PCOS risk [4-7], that obesity and PCOS are causally related. Since the mechanism(s) by which FTO variants affect fat mass are not currently known, other possible causal relationships (such as a direct effect of FTO variants on the development of PCOS, independent of the effects on fat mass) cannot be excluded, although a distinct non-adiposity effect of FTO variants on PCOS susceptibility would seem unlikely. Elucidation of the biological role and mechanism(s) of action of FTO variants with respect to weight and obesity should help to confirm how they influence PCOS susceptibility.

In summary, we have shown that variants in FTO which are known to influence the metabolic profile through effects on fat mass, BMI and obesity [24], and which are known to affect the risk of type 2 diabetes mellitus, are also associated with PCOS in UK women. The most likely explanation for this finding is an indirect effect on PCOS risk, mediated through an effect on fat mass and resulting in deleterious metabolic consequences. This would be consistent with the data [24] that indicate that the additional fat mass associated with FTO variation exerts metabolic effects which are in line with the epidemiologically defined relationships between fat mass and direct and indirect measures of insulin resistance. FTO is one of the first genes for which a robust effect of genome sequence variation on PCOS risk can be claimed. Whether or not FTO can be labelled as a PCOS susceptibility gene as opposed to a gene that purely influences fat mass is essentially a semantic argument, analogous to that regarding the status of FTO as a susceptibility gene for type 2 diabetes mellitus.

Acknowledgements This work was funded by grants and fellowships from the Wellcome Trust (GR069224MA, 068545/Z/02), the UK Medical Research Council (G9700120, G0000934), the Academy of Finland (M.-R. Järvelin) and NovoNordisk (Clinical Research Fellowship to T. M. Barber). We acknowledge the many patients, relatives, nurses and physicians who contributed to the ascertainment of the various clinical samples used in this study, and the WTCCC.

Duality of interest The authors declare that there is no duality of interest associated with this manuscript.

\section{References}

1. Asuncion M, Calvo RM, San Millan JL, Sancho J, Avila S, Escobar-Morreale HF (2000) A prospective study of the prevalence of the polycystic ovary syndrome in unselected Caucasian women from Spain. J Clin Endocrinol Metab 85:2434-2438

2. Legro RS (2000) The genetics of obesity. Lessons for polycystic ovary syndrome. Ann N Y Acad Sci 900:193-202

3. Balen AH, Conway GS, Kaltsas G et al (1995) Polycystic ovary syndrome: the spectrum of the disorder in 1741 patients. Hum Reprod 10:2107-2111

4. Kiddy DS, Hamilton-Fairley D, Bush A et al (1992) Improvement in endocrine and ovarian function during dietary treatment of obese women with polycystic ovary syndrome. Clin Endocrinol $36: 105-111$

5. Holte J, Bergh T, Berne C, Wide L, Lithell H (1995) Restored insulin sensitivity but persistently increased early insulin secretion after weight loss in obese women with polycystic ovary syndrome. J Clin Endocrinol Metab 80:2586-2593

6. Taponen S, Martikainen H, Jarvelin MR et al (2003) Hormonal profile of women with self-reported symptoms of oligomenorrhea and/or hirsutism: Northern Finland birth cohort 1966 study. J Clin Endocrinol Metab 88:141-147

7. Barber TM, McCarthy MI, Wass JA, Franks S (2006) Obesity and polycystic ovary syndrome. Clin Endocrinol 65:137-145

8. Walley AJ, Blakemore AI, Froguel P (2006) Genetics of obesity and the prediction of risk for health. Hum Mol Genet 15(Spec No 2):R124-130

9. Vink JM, Sadrzadeh S, Lambalk CB, Boomsma DI (2006) Heritability of polycystic ovary syndrome in a Dutch twin-family study. J Clin Endocrinol Metab 91:2100-2104

10. Franks S (1995) Polycystic ovary syndrome. N Engl J Med 333:853-861

11. Urbanek M (2007) The genetics of the polycystic ovary syndrome. Nat Clin Pract Endocrinol Metab 3:103-111

12. Bell CG, Walley AJ, Froguel P (2005) The genetics of human obesity. Nat Rev Genet 6:221-234

13. Frayling TM, Timpson NJ, Weedon MN et al (2007) A common variant in the FTO gene is associated with body mass index and 
predisposes to childhood and adult obesity. Science 316:889894

14. Dina C, Meyre D, Gallina S et al (2007) Variation in FTO contributes to childhood obesity and severe adult obesity. Nat Genet 39:724-726

15. The Rotterdam ESHRE/ASRM-sponsored PCOS consensus workshop group (2004) Revised 2003 consensus on diagnostic criteria and long-term health risks related to polycystic ovary syndrome (PCOS). Hum Reprod 19:41-47

16. Adams J, Polson DW, Franks S (1986) Prevalence of polycystic ovaries in women with anovulation and idiopathic hirsutism. BMJ (Clin Res Ed) 293:355-359

17. Barber TM, Bennett AJ, Gloyn AL et al (2007) Relationship between E23K (an established type II diabetes-susceptibility variant within $\mathrm{KCNJ} 11$ ), polycystic ovary syndrome and androgen levels. Eur J Hum Genet 15:679-684

18. Powell BL, Haddad L, Bennett A et al (2005) Analysis of multiple data sets reveals no association between the insulin gene variable number tandem repeat element and polycystic ovary syndrome or related traits. J Clin Endocrinol Metab 90:2988-2993
19. Rantakallio P (1988) The longitudinal study of the northern Finland birth cohort of 1966. Paediatr Perinat Epidemiol 2:59-88

20. Taponen S, Ahonkallio S, Martikainen H et al (2004) Prevalence of polycystic ovaries in women with self-reported symptoms of oligomenorrhoea and/or hirsutism: Northern Finland Birth Cohort 1966 Study. Hum Reprod 19:1083-1088

21. Taponen S, Martikainen H, Jarvelin MR et al (2004) Metabolic cardiovascular disease risk factors in women with self-reported symptoms of oligomenorrhea and/or hirsutism: Northern Finland Birth Cohort 1966 Study. J Clin Endocrinol Metab 89:2114-2118

22. Colhoun HM, McKeigue PM, Davey Smith G (2003) Problems of reporting genetic associations with complex outcomes. Lancet 361:865-872

23. Scuteri A, Sanna S, Chen WM et al (2007) Genome-wide association scan shows genetic variants in the FTO gene are associated with obesity-related traits. PLoS Genet 3:e115

24. Freathy RM, Timpson NJ, Lawlor DA et al. (2008) Common variation in the FTO gene alters diabetes-related metabolic traits to the extent expected, given its effect on BMI. Diabetes DOI $10.2337 / \mathrm{db} 07-1466$ 